\title{
Research on acquisition and feature analysis of GMA status signal based on grating sensors
}

\author{
Ping $\operatorname{Han}^{1, a}$ and Kaichong $\mathrm{Ma}^{1, \mathrm{~b},{ }^{*}}$ \\ ${ }^{1}$ School of Information and Engineering, Wuhan University of Technology, Wuhan 430070, China; \\ ahanping@whut.edu.cn, b398164148@qq.com \\ ${ }^{*}$ Correspondence author
}

Keywords: Giant Magnetostrictive Actuator; BP neural network; Grating sensors.

\begin{abstract}
The new GMA experimental platform based on grating sensors and modeling method with BP neural network are proposed. The various parameters of GMA in the working process, such as driving voltage, prestress, displacement, are gathered and the effects are analyzed, then BP neural network is used to build the GMA model according with the real features of driving voltage and prestress. The model is verified by experiment with actual data, it can predict the actuating output of GMA greatly, and the predict error is in the range of $0-0.004 \mathrm{~nm}$.
\end{abstract}

\section{Introduction}

In the actuating process of Giant Magnetostrictive Actuator (GMA), nonlinear characteristics of micro-actuated structure can be changed by the coupling of different physical field factors such as excitation voltage, frequency, magnetic field intensity, prestress, temperature, etc. Therefore, GMA nonlinear characteristic modeling is a hotspot in the present study. It points out that the prestress can affect the size of the magnetostrictive strain, but there are very few collected data and the status signals are not highly precise ${ }^{[1]}$. The driving frequency has loss effect on GMA, but it failed to give the direct relationship between the frequency and output displacement ${ }^{[2]}$. Bias magnetic field affects the efficiency of Giant Magnetostrictive Material(GMM), coupling coefficient, permeability and other coefficient, but the data acquisition system is too complicated ${ }^{[3]}$. It shows that temperature would cause thermal deformation of GMM rod, shell and other metal materials and which also have impact on giant magnetostrictive coefficient, but there is no detailed analysis on the accurate relationship between temperature change and GMA displacement ${ }^{[4]}$.

According to the deficiency of the above research, this paper sets up a GMA experimental platform based on grating sensors, makes acquisition and analysis on each status parameter in the working process of GMA, and conducts dynamic modeling of GMA, and the characteristic simulation and analysis for the model are made with Matlab.

\section{GMA experimental platform}

The experimental platform structure is shown in figure $2-1(a)^{[5]}$. Signal source comes to GMA through the power amplifier, then GMM rod will have displacement which causes the variant to bend and the pitch of grating sensor pasted above to change, and it will result in changes of wavelength. Then the signal will be sent to computer through grating demodulator.

As shown in figure 2-1(b), two gratings are pasted to GMA device of this experiment. The first grating is firmly fixed on a variant with glue, which is trying to keep the fiber bragg grating straight and parallel to the variant. The variant adopted by this device is steel rule, and the steel ruler is fixed above the GMA. When the current is injected into GMA coil, it will cause the expansion of the telescopic rod and compress the steel ruler and make it bend, then lead the strain of the fixed grating sensor. Thus the data about wavelength change is collected from the demodulator. The second grating sensor is pasted close to GMM rod, when GMM vibration occurs, bragg wavelength of the grating sensor on GMM rod will change, thus the vibration data of GMM rod is collected. 


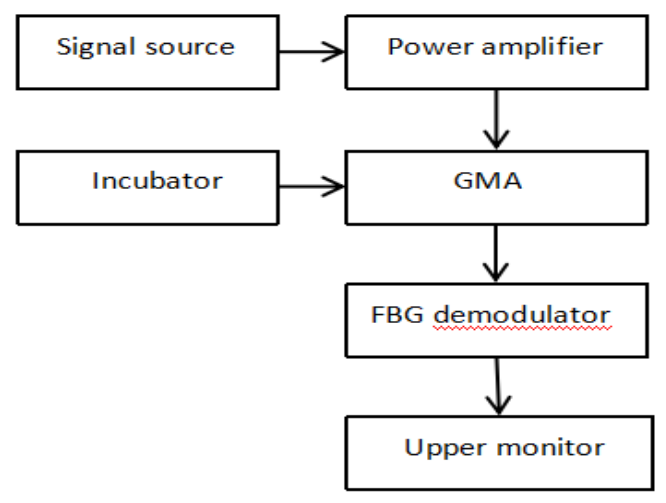

Fig.2-1 (a)GMA experimental platform

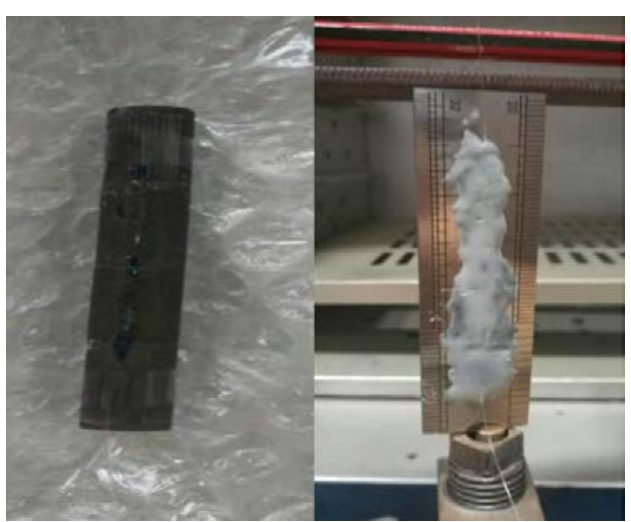

(b)paste grating sensor to GMM and variant

\section{Parameters measurement}

Considering the experimental environment and the precision of the experiment device, control variable method is chosen to be the main method to measure the data for this experimental.

When debugging range of signal source peak-to-peak voltage is from $2.0 \mathrm{~V}$ to $0.6 \mathrm{~V}$, the changes of the waveform are obvious and never exceed the maximum withstand voltage of GMA. The range of corresponding peak-to-peak voltage amplified by power amplifier is from $2.4 \mathrm{~V}$ to $8.0 \mathrm{~V}$ with step of $0.8 \mathrm{~V}$. There are 8 sets of data in total. When measuring the prestress characteristics of GMA, prestress is reflected by the angle of rotating pre-tightening screw. After many times of authentication, when the angle of pre-tightening screw is $20^{\circ}$, the prestress will be the minimum; When the screw is turned to $40^{\circ}$, the prestress will be the maximum. Therefore, the pre-tightening screw adjusting range is from $20^{\circ}$ to $40^{\circ}$ with step of $5^{\circ}$, there are 5 sets of data in total. The Butterworth band-pass filter is adopted to filter the experimental data, and the program is shown in 3-1:

$$
[B, A]=\operatorname{BUTTER}\left(N, W_{n}\right)
$$

Wherein, $n$ is filtering order, $W_{n}$ is normalized cut-off frequency which is mainly related to sampling frequency. After repeated verification, when $n=1$, the minimum normalized cut-off frequency is $1 / 26$, and the maximum normalized cut-off frequency is $1 / 13$, the filtering effect is good, and the program is shown in 3-2.

$$
\begin{aligned}
& {[b, a]=\text { butter(1,[1/26,1/13]); }} \\
& \text { test = filtfilt(b, a, signal); }
\end{aligned}
$$

\section{Neural network modeling of GMA}

Now there are three models that mainly used for characteristics analysis ${ }^{[6]}$. They are Jiles-Atherton model, Preisach model and neural network model. Preisach model needs to distinguish many non-physical parameters which are easy to produce high order equation. Secondly, Preisach model can get corresponding inverse model only through mathematical calculation, so it is difficult to realize real-time compensation and control. The analysis on coupling feature by Jiles-Atherton model is not comprehensive enough, it only pays attention to the magnetic model in quasi state without considering the influence of frequency. However, neural network model has this good nonlinear function fitting ability, self-adaptation and self-learning ability as well as fault tolerance ability ${ }^{[7]}$. So that the model can accurately analyze the characteristic of various parameters of GMA. The neural network modeling structure of this experiment is shown in Figure 4-1. 


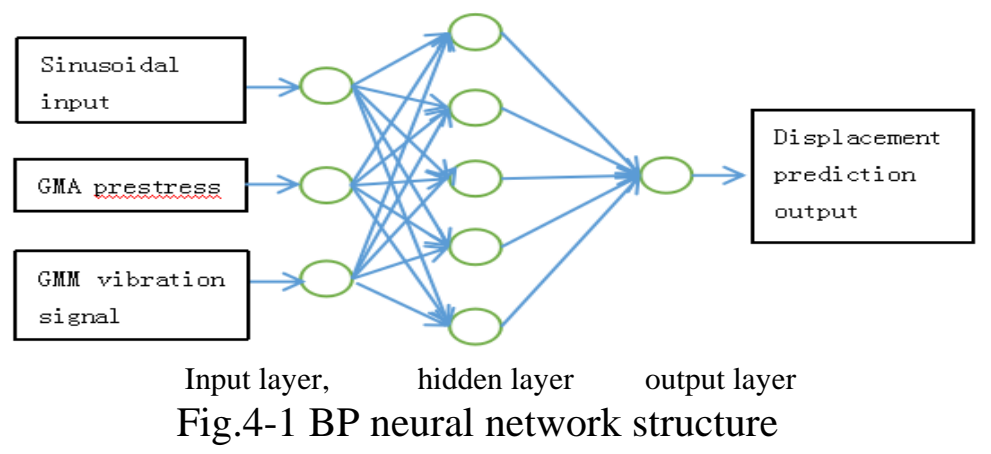

\subsection{BP neural network model of drive voltage}

The range of driving voltage in this experiment is from $2.4 \mathrm{~V}$ to $8.0 \mathrm{~V}$. In order to control the temperature, the whole experiment was conducted in an incubator with temperature of $25^{\circ}$, frequency of $100 \mathrm{hz}$ and the angle of pre-tightening screw is $40^{\circ}$ which remain unchanged. There are 8 sets of waveform data that selected from different voltage as below. 1000 data is selected from each set. It comes out 8000 data in total. With the abscissa as count and ordinate as actuator output displacement. The displacement output waveform under driving voltage of $2.40 \mathrm{~V}, 3.20 \mathrm{~V}, 4.00 \mathrm{~V}, 4.80 \mathrm{~V}, 5.60 \mathrm{~V}$, $6.40 \mathrm{~V}, 7.20 \mathrm{~V}$ and $8.00 \mathrm{~V}$ is shown in Figure $4-1$ from left to right.

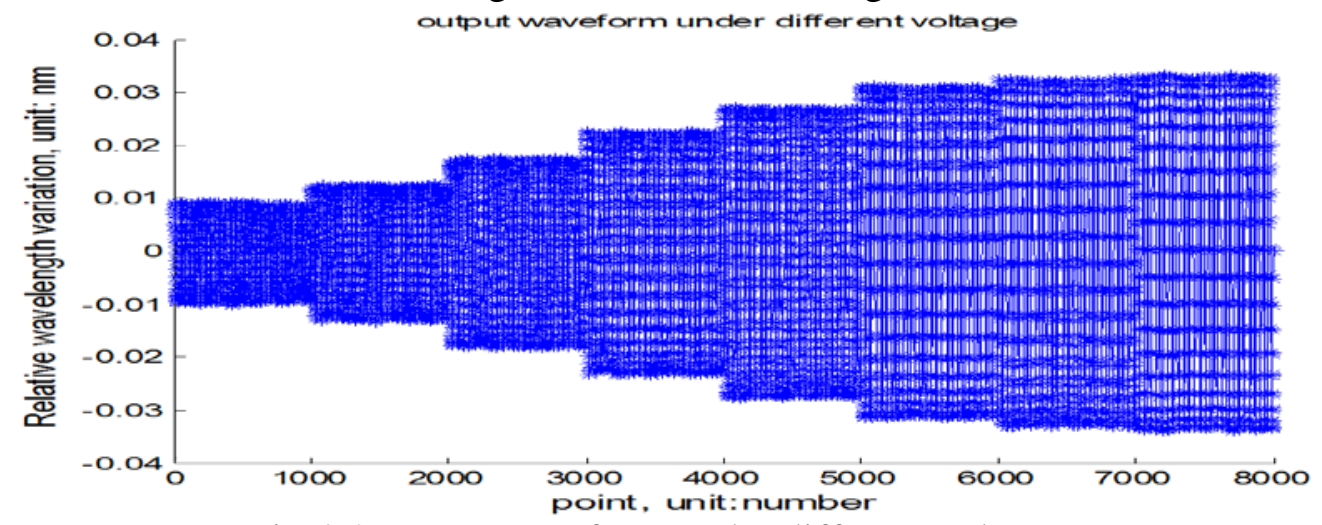

Fig.4-2 output waveform under different voltage

In order to improve the accuracy of modeling, 3000 data that relatively stable from each data set after filtering is selected to form into a waveform as the training sample of 24000 data. Then 1000 data is selected from the residual data of each set after filtering to form a waveform as predicted sample of 8000 data. Before the model training, generally the training data need to be treated. Filtering treatment is first given to waveform data in this paper and then normalized is provided for all waveform data with mapminmax function in MATLAB R2012b software to make all of them fall into the interval of $[1,1]$.

Driving voltage signal, GMA prestress and GMM vibration signal are the input of this experiment, the output is GMA displacement signal, hence the input node is 3 and the output node is 1 . In order to save training time, the single hidden layer is chosen under such premise. Ansig function is selected as the transfer function of hidden layer, logsig function is the transfer function of the output layer. Learning rate and the target parameter value are set to be 0.1 and 0.0004 . With 24000 voltage data being used for training and the remaining 8000 data as prediction, the prediction waveform is shown in figure 4-3(a) as below, and error graph of predicted output and desired output is shown in figure 4-3(b). 

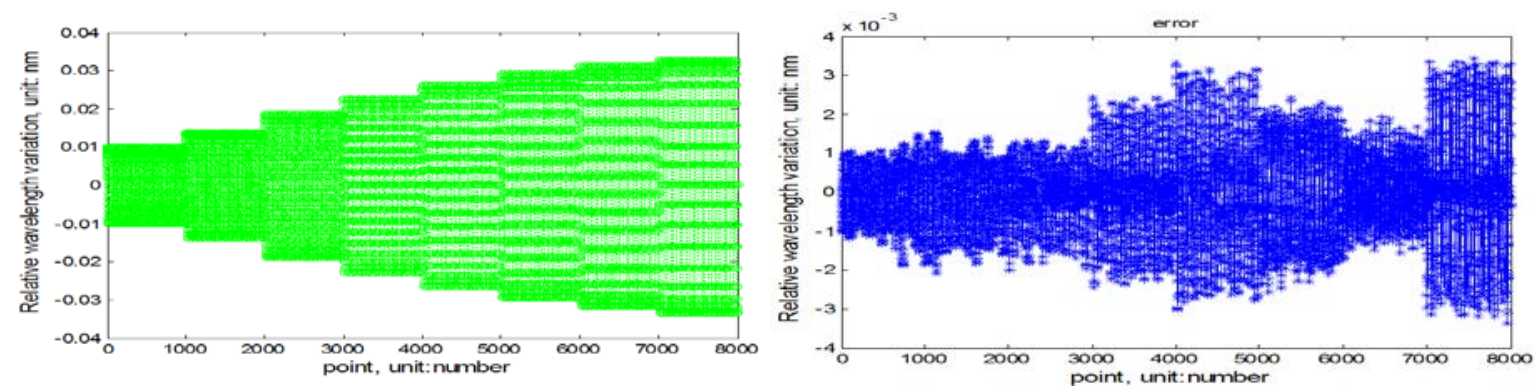

Fig.4-3(a) output waveform under different voltage

(b) output error

Compared with actual output, predicted output has higher waveform fitting degree, which indicates the model has good predicting outcomes.

\subsection{BP neural network model of prestress}

In this prestress experiment, control variable method is used to keep drive voltage as $8 \mathrm{v}$, frequency as $100 \mathrm{hz}$, and temperature as $25^{\circ} \mathrm{C}$. The size of prestress is changed only. 5 sets of output waveform data that measured under $20^{\circ}, 25^{\circ}, 30^{\circ}, 35^{\circ}$ and $40^{\circ}$ were selected from it. 1000 data is selected from each set, 4000 data comes out in total. With the abscissa as count and ordinate as actuator output displacement. The output waveform of pre-tightening screw under $20^{\circ}, 25^{\circ}, 30^{\circ}, 35^{\circ}$ and $40^{\circ}$ is shown from left to right in Figure 4-4 .

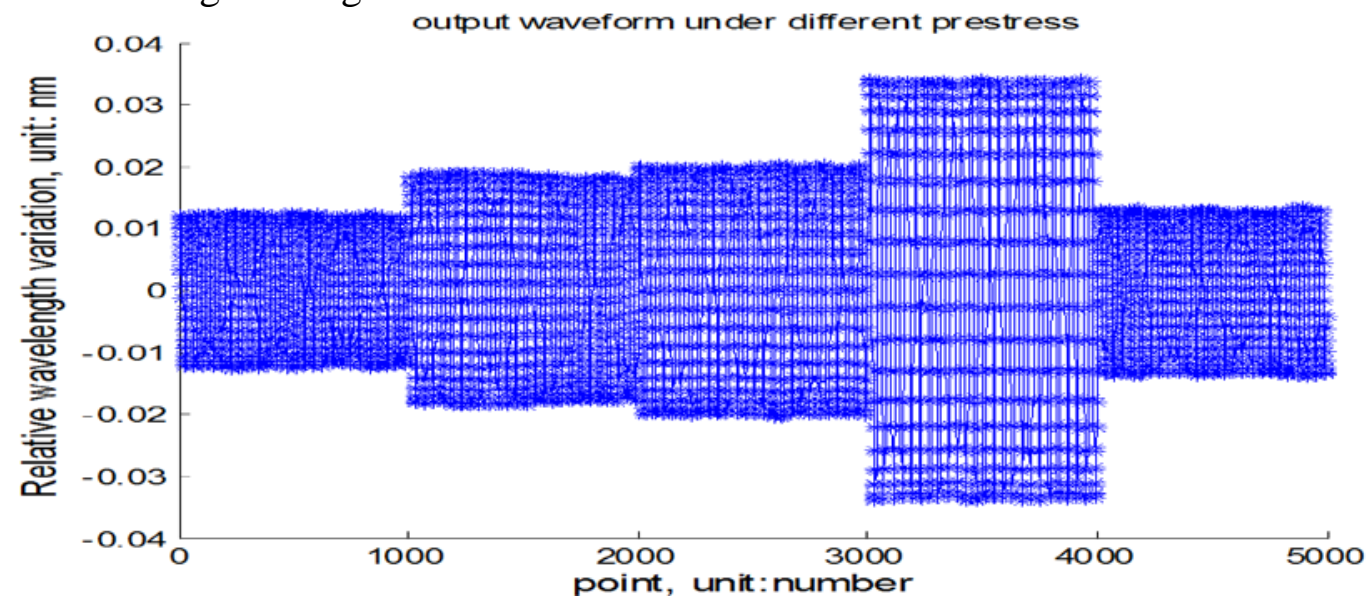

Fig.4-4 output waveform under different prestress

In order to guarantee the comparability of the model output results, the prestress model network structure is the same as the drive voltage that is single hidden layer and multiple nodes for setting parameters of this prestress model. Except different selection of data, the selection of number of input and output nodes of the network, transfer function, performance function and learning function are the same as drive voltage model network structure. With 15000 voltage data as the training, the remaining 50000 data as prediction, the predicted waveform is shown in figure 4-5(a), and the error of predicted output and desired output is shown in figure 4-5(b):

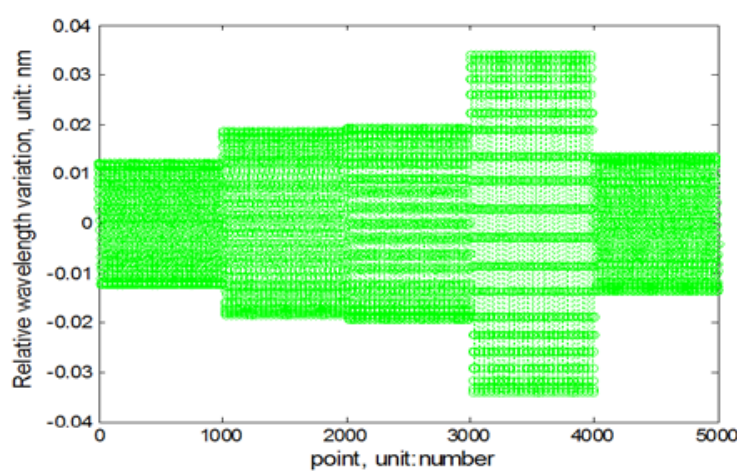

Figure.4-5(a) output waveform under different prestress

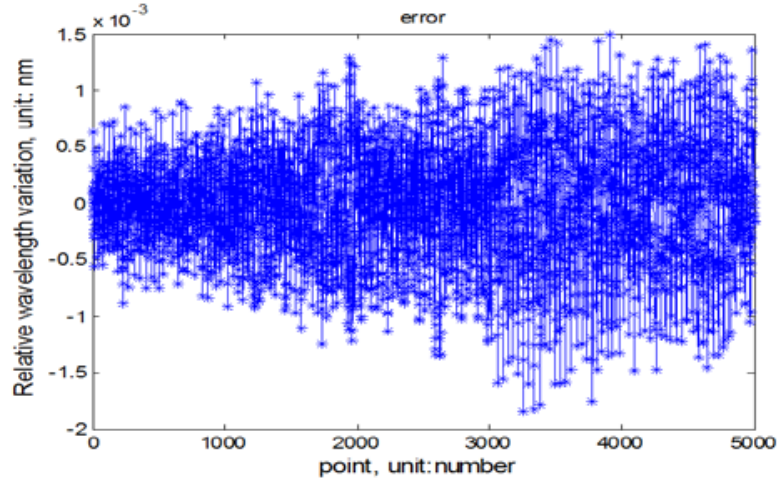

(b) output error

Compared with actual output, predicted output has higher waveform fitting degree, which indicates the model has good predicting outcomes.

\subsection{Characteristic analysis}


According to the above that neural network modeling verifies the change of drive voltage and prestress affects the output displacement of GMA. That shows some certain regularities:

(1) In a certain range (voltage gradually increases from $2.4 \mathrm{~V}$ to $7.2 \mathrm{~V}$ ), when drive voltage increases, the output displacement of GMA increases.

(2) When the voltage is greater than $7.2 \mathrm{~V}$, with the increase of voltage, the output displacement of GMA increases slowly and gradually tends to be stable.

(3)When the torsion angle of pre-tightening screw ranging from $20^{\circ}$ to $35^{\circ}$, with the increase of prestress, GMA output displacement increases.

(4)When the torsion angle of pre-tightening screw is more than $35^{\circ}$, GMA output displacement suddenly decreases and is lower than the displacement when pre-tightening screw is at $20^{\circ}$.

It shows that influence of drive voltage on GMA is unitary. The change trend is a process that from gradual increase to slow increase till being stable, while the influence trend of prestress on displacement of GMA is a process from gradual increase to gradual decrease, if prestress is too small, it is unable to give full play to the performance advantage of GMA. That causes the displacement output of GMA to be worse. So that choosing appropriate prestress has a great influence on output performance of GMA. In this experiment, GMA has the maximum displacement output when the angle of pre-tightening screw is 35 degrees.

\section{Summary}

With GMA as the research object of this paper, according to two influence factors drive voltage and prestress, firstly bulk data measurement and early treatment are carried out. Then BP neural network modeling is conducted based on the data obtained. It makes characteristic analysis for these two influence factors. The error curve shows that model has certain accuracy. For future work, the next step of this paper will be studying the coupling relation between drive voltage and prestress and making modeling analysis next.

\section{Acknowledgements}

This paper is supported by National Natural Science Foundation of China(51405360).

\section{References}

[1]. Taotao Li: The Research and Analysis of High Frequency Vibration Performance of Giant Magnetostrictive Actuator (Master Degree of Engineering, Wuhan University of Technology, China, 2014).

[2]. Liyi Li, Baiping Yan, Chengming Zhang. Influence of Frequency on Characteristic of Loss and Temperature in Giant Magnetostrictive Actuator. Proceedings of the CSEE. Vol.31(2011) No.18.

[3]. Wei Wang: Research on High Stability Giant Magnetostrictive Micro-Displacement Actuator (Master Degree of Engineering, Harbin Institute of Technology , China, 2009).

[4]. Tingxin Ming, Menglun Tao, Zhiwei Huang. The Design and Analysis of the Temperature Control System of Giant Magnetostrictive Actuator.Journal of Wuhan University of Technology. Vol.37(2015) No.9.

[5]. Menglun Tao: Structure Design and Property Research of Giant Magnetostrictive Actuators (Master Degree of Engineering, Wuhan University of Technology, China, 2008).

[6]. Shuying Cao: Dynamic Model With Hysteresis Nonlinearity and Control Technique for Giant Magnetostrictive Actuator (Doctor Degree of Engineering, Hebei University of Technology, China, 2004).

[7]. Shuying Cao, Jiaju Zheng, Bowen Wang. Precision Position Control for Giant Magnetostrictive Actuator Based on Dynamic Recurrent Neural Network. Proceedings of the CSEE. Vol. 26 (2006) No. 3. 